\title{
Pneumocystis Jirovecii Pneumonia: Molecular Diagnosis and Antimicrobial Sus- ceptibility in Two Renal Transplants Cases
}

\author{
Baccouchi Nawel ${ }^{1,2, *}$, Mtibaa Latifa ${ }^{2}$, Souid Hana ${ }^{1,2}$, Kacem Imen ${ }^{3}$, Selmi Yosra ${ }^{3}$, Ben Rhomdhane Nabil ${ }^{3}$, and Jemli Boutheina ${ }^{2}$ \\ ${ }^{1}$ Faculty of Sciences, Tunis El-Manar, Tunis, Tunisia \\ ${ }^{2}$ Laboratory of Parasitology \& Mycology, Military Hospital of Tunis, 1008 Monfleury, Tunis, Tunisia \\ ${ }^{3}$ Organ Transplant Unit, Military Hospital of Tunis, 1008 Monfleury, Tunis, Tunisia
}

*Corresponding authors: Baccouchi Nawel, Faculty of Sciences, Tunis El-Manar, Tunis, Tunisia, Tel: 27186634-50812314; E-mail address: nawel.baccouchi@ fst.utm.tn

Received: 20 Apr, 2021 | Accepted: 04 May, 2021 | Published: 11 May, 2021

Citation: Nawel B, Latifa M, Hana S, Imen K, Yosra S, et al. (2021) Pneumocystis Jirovecii Pneumonia: Molecular Diagnosis and Antimicrobial Susceptibility in Two Renal Transplants Cases. J Clin Case Stu 6(2): dx.doi.org/10.16966/2471-4925.220

Copyright: (C) 2021 Nawel B, et al. This is an open-access article distributed under the terms of the Creative Commons Attribution License, which permits unrestricted use, distribution, and reproduction in any medium, provided the original author and source are credited.

\section{Abstract}

Objectives: Pneumocystis jirovecii, a pathogenic fungus, causes severe interstitial pneumonia among immunocompromised patients. The transplant patients are predisposed to $P$. jirovecii pneumonia (PJP), most notably those under immunosuppressive therapy or with graft rejection. We describe here two cases of renal transplant cases with PJP and demonstrate the importance of molecular biology in the diagnosis and the prevalence of the DHPS gene mutation.

Material and methods: Diagnosis of Pneumocystis jirovecii was made using conventional techniques: direct examination with staining GomoriGrocott modified by MUSTO (GG), May-Grünwald Giemsa (MGG), and Direct immunofluorescence (DIF) MONOFLUO ${ }^{\text {TM }}$ Pneumocystis jirovecii IFA Test Biorad. Molecular diagnosis was done with real-time PCR (Liferiver ${ }^{\mathrm{TM}}$ Pneumocystis jirovecii ${ }^{\oplus}$ ), and polymerase chain reaction-restriction fragment length polymorphism (PCR-RFLP).

Results: For the two samples, MGG, GG, and DIF staining did not allow the detection of a cystic or vegetative form. Real-time PCR reveals positive results for both samples for a quantity of DNA 103 copies per milliliter and 104 copies per milliliter. Amplification of the DHPS gene shows that no mutation has occurred in this gene. An improvement in the general condition of patients after 14 days of treatment with Sulfamethoxazole/ Trimethoprim was noted.

Conclusions: Our study shows that PCR is a faster and more sensitive technique than staining techniques. Currently, real-time PCR offers both a quantitative and qualitative approach with a very low detection threshold which makes it possible to differentiate between simple colonization and infection.

Keywords: Pneumocystis jirovecii; Pneumonia; Real-time PCR; Renal transplant

\section{Introduction}

Pneumocystosis is an opportunistic mycosis caused by Pneumocystis jirovecii, a nubiquitous eukaryotic microorganism, classified among fungi in 1980 [1]. Up to $45 \%$ of human immunodeficiency virus (HIV) patients suffer from Pneumocystis pneumonia (PJP). In addition, PJP cases in non-HIV-patients are increasing due to the use of glucocorticoids, chemotherapy, or other immunosuppressive drugs [1-3]. Furthermore, it is an increasing problem in transplanted patients: in the USA, up to $25 \%$ of these patients suffer from a PJP [3].

The microbiological diagnosis of the PJP is usually based on microscopic demonstration of the fungus in the respiratory specimens by staining methods. The development of molecular biology techniques has significantly improved the diagnosis [4-6]. Molecular methods show more sensitivity and specificity compared to microbiological methods $[7,8]$. Furthermore, by quantitative PCR
(qPCR), the quantity of the fungus in a specimen and even the status of the patient can be determinate $[4,9,10]$.

The prophylaxis and the treatment of this disease are based on sulfonamides especially trimethoprim-sulfamethoxazole combination (TMP-SMX) or cotrimoxazole. As result, cases of resistance were selected with a mutation at the 'dihydropteroate synthase (DHPS)', the target of sulfonamides $[11,12]$.

We describe here two cases of renal transplant patients with PJP and demonstrate the importance of molecular biology in the diagnosis and the prevalence of the DHPS gene mutation.

\section{Methods}

\section{Case description}

Case 1: A 53-year-old woman, suffering from renal failure caused by polycystic kidney disease, with-renal transplant in 2016 with chronic 
rejection under Mycophenolate Mofetil-tacrolimus-Prednisolone sodium metasulfobenzoate. She was admitted for an altered general condition consisting of asthenia, anorexia, fever of $39^{\circ} \mathrm{C}$, and polypnea. Laboratory data showed C reactive protein $(\mathrm{CRP})$ of $355 \mathrm{mg} / \mathrm{ml}$, $\mathrm{Hb}=9.2 \mathrm{~g} / \mathrm{dl}, \mathrm{WBC}=7800 / \mathrm{mm}^{3}, \mathrm{PLT}=181000 / \mathrm{mm}^{3}$, urea $=14.5 \mathrm{mmol} / \mathrm{l}$, creatinine $=303 \mu \mathrm{mol} / \mathrm{l}, \mathrm{K}=4.2 \mathrm{mmol} / \mathrm{l}$, serum calcium $=2.53 \mathrm{mmol} / \mathrm{l}$. Chest X-Ray showed bilateral alveolar-interstitial syndrome. Serology for Candida spp and Aspergillus spp was negative; search for BK in the sputum was negative. Cytomegalovirus (CMV) PCR was negative. Microbiological investigation showed a urinary tract infection by Escherichia coli and a positive $P$. jirovecii real-time PCR. The patient was initially put on Tazocillin-Ciprofloxacin-Sulfamethoxazole/ Trimethoprim, then on Ceftazidime and Sulfamethoxazole/ Trimethoprim. General condition after one week with a second negative $P$. jirovecii PCR.

Case 2: A man, 62 years old with-kidney transplant since 2002. Graft biopsy puncture in 2018 showed idiopathic extra membranous glomerulonephritis which recurred when put on Cyclosporine and a high dose of corticosteroid. The clinical examination on admission noted a fever of $38^{\circ} \mathrm{C}$ and edema of the lower limbs. The chest X-Ray showed an alveolar-interstitial syndrome.

Serology for Candida spp and Aspergillus spp were negative. Search for BK in urine and sputum was negative. Blood and urine cultures were negative. CMV PCR was positive with 1136 copies $/ \mathrm{mL}$. Also, PCR for P. jirovecii was positive. The patient was treated on Cymevan, Augmentin, and Sulfamethoxazole/Trimethoprim, with a reduction of the dose of immunosuppressive drugs.

The evolution was marked by improvement in the general condition after 14 days with a second PCR P. jirovecii negative.

\section{Microbiological methods}

Respiratory specimens (sputum for both patients) were centrifuged at $6000 \mathrm{~g}$ for $10 \mathrm{~min}$. Part of the suspended pellet (about 200 $\mu$ ) was used for microscopic examination (MGG, GG stains) and $200 \mu$ was used for real-time PCR for detection and quantification of $P$. jirovecii DNA and PCR-RFLP for the detection of DHPS gene mutations in $P$. jirovecii strains.

\section{Conventional techniques-microscopy}

The MGG stain highlights intra-cystic bodies and/or trophozoites of $P$. jirovecii. Giemsa stains the nuclei of all Pneumocystis life cycle stages in purple-pink but does not stain the cell wall while GG stains the wall of cysts of $P$. jirovecii in black brown on a green background.

The DIF using the kit MONOFLUO ${ }^{\text {ix }}$ Pneumocystis jirovecii IFA Test (Biorad) allows highlighting the presence of pathogen (cyst or vegetative form) by immunological labeling. The attachment of the pathogen to the fluorescein-labeled antibody gives an apple-green coloring according to manufacturer's instructions for use.

\section{Molecular diagnosis}

Real-time PCR using the Life river ${ }^{\mathrm{Tm}}$ Pneumocystis jirovecii ${ }^{\circledast}$ allows the detection and quantification of $P$. jirovecii DNA. PCR-RFLP was used for DHPS locus analyzes.

\section{Results}

For two samples, the MGG and GG staining did not allow the detection of a cystic or vegetative form. The DIF technique also remains negative. Real-time PCR reveals a positive result for two samples 103 copies per milliliter and 104 copies per milliliter respectively.
(Figure 1) showed the quantification of $P$. jirovecii DNA. (Figure 2) illustrates the amplification of the DHPS gene at 370bp with conventional PCR. PCR RFLP shows that no mutation has occurred in this gene for the two cases (Figure 3)

An improvement in the general condition of patients after 14 days of treatment with Bactrim ${ }^{\circ}$ was noted. Even real-time PCRs for $P$. jirovecii after this interval are negative.

\section{Discussion}

Currently, cases of pneumocystosis in HIV-infected patients tend to decrease due to the use of anti-Pneumocystis jirovecii chemoprophylaxis $[1,4,13]$. Nevertheless, its incidence has dramatically increased over the last 15 years in patients with other predisposing immunodeficiencies [1], particularly transplant recipients [14-17]. In our study, we diagnosed the presence of $P$. jirovecii in two renal transplant patients, non-HIV. Magne, et al. reported 805 cases of PJP including 264 (33\%) non-HIV, 86 had undergone a transplant among them 37 kidney transplantation [18].

In our study, case 1 has a notion of chronic rejection. Case 2 has a CMV infection. The median time from transplantation to the development of disease was 18 months for case 1 and 16 years for case 2. Several risk factors have been identified as predisposing transplant patients to PJP, most notably in immunosuppressive therapy, graft rejection, CMV infection, and older age $[3,19]$. Bostnar, et al. reported that $13 / 601(2.2 \%)$ of transplant patients have developed PJP. The median time from the transplantation to the development of disease was 17 months [4]. Three patients had PJP during the first year after transplantation, three had graft rejection, and six had a CMV infection. New studies indicate that high-dose immunotherapy with a long course of corticosteroids and treatment with anti-lymphocyte antibodies present special risk factors for PJP after renal transplantation [20].

PJP is particularly characterized by dry cough with progressive dyspnea, producing a hypoxemic interstitial pneumopathy often bilateral developing in a febrile context [6]. Our patients suffer from fever, cough, and alveolar-interstitial syndrome.

Real-time PCR was positive for the two samples while no cyst and/or trophozoite was detected by conventional techniques. The sensitivity of the staining techniques was acceptable (89-98\%) for bronchoalveolar lavage samples but low (35-78\%) for aspiration and sputum samples $[1,5]$. In the Bossart, et al. study, all 31 PCRs were positive, of which 30 were positive for DIF. The only patient with the discordant result had chronic obstructive pulmonary disease [4]. The lack of sensitivity of microscopic methods is explained by the difficulty of reading when samples are non-invasive or superficial. Indeed, the introduction of molecular biology techniques in the diagnosis of PJP increased the detection sensitivity of $P$. jirovecii (86-100\%) whatever the sample bronchoalveolar lavage, aspirates, or sputum samples [6]. The main advantage of real-time PCR is the simultaneous performance of the amplification and detection steps in a closed system, which reduces the risk of contamination. Real-time PCR positive controls also have a well-defined quantification $\left(10^{7} \mathrm{ml}^{-1}\right.$ copies $)$. Moreover, it allows DNA to be detected even in small quantities to be able to specify whether it is simple colonization or infection and therefore to determinate patient's status $[5,4]$.

The emergence of $P$. jirovecii drug resistance has been suggested recently by the mutations in the gene encoding dihydropteroate synthase (DHPS). Jarboui, et al. revealed that for the 21 immunocompromised patients, only 17 (81\%) were positive by DHPS amplification. Only 
I

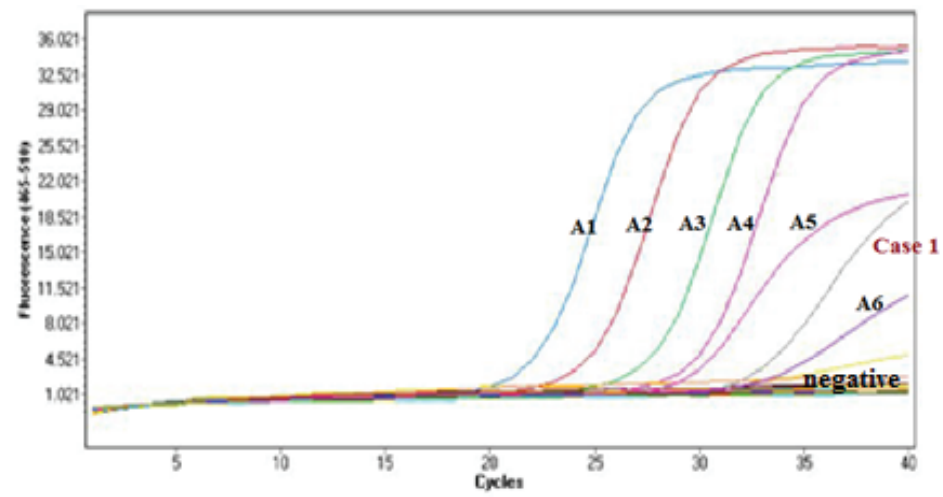

II

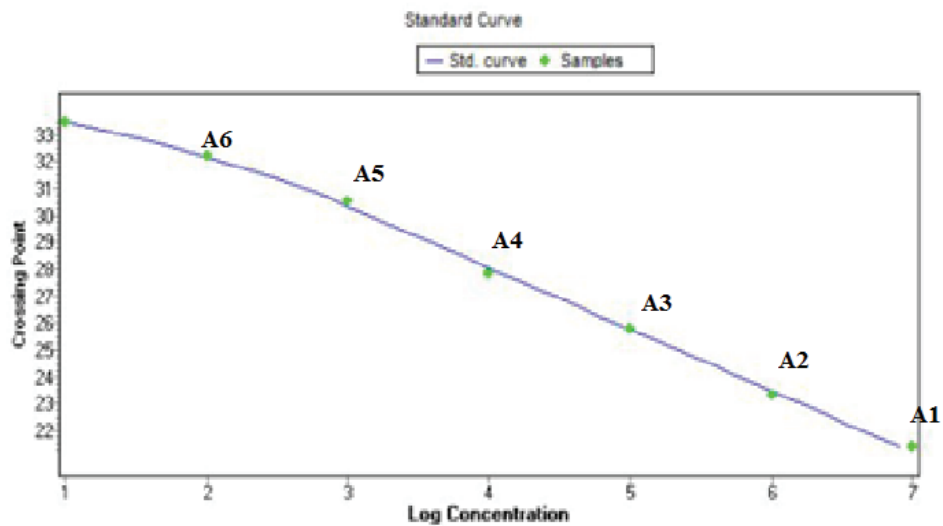

Figure 1: The curve of the quantification of PJ DNA by real-time PCR.

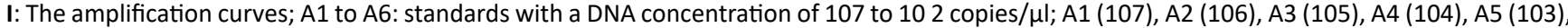
A6 (102), Case 1: the sample tested; Negative: negative control.

II: The standard curve constructed from the values of $\mathrm{Cp}$ (crossing point) versus the log DNA copy number/(range=107 to $102 \mathrm{copies} / \mu \mathrm{l})$.

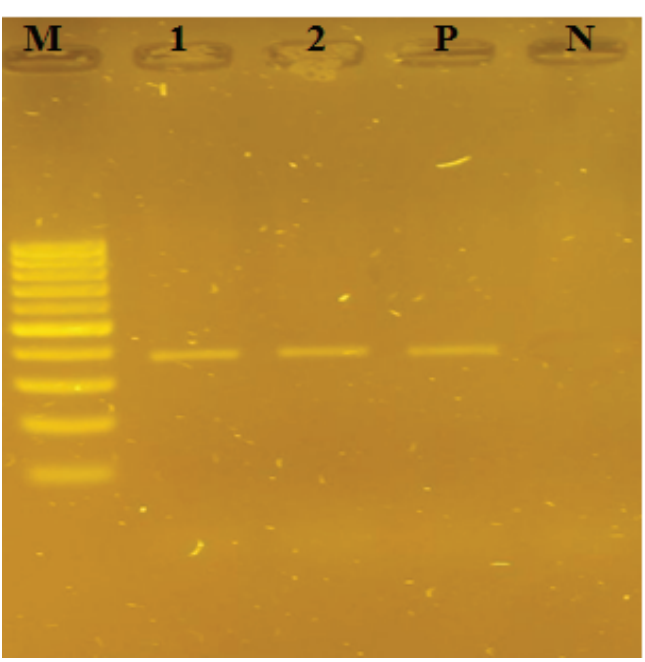

Figure 2: Agarose gel electrophoresis of conventionnal PCR products of $P$. jirovecii DHPS gene $370 \mathrm{pb}$.

Lanes: M (100 bp DNA size marker); 1 (Case 1); 2(Case 2); P (positive control); $\mathrm{N}$ (negative control).

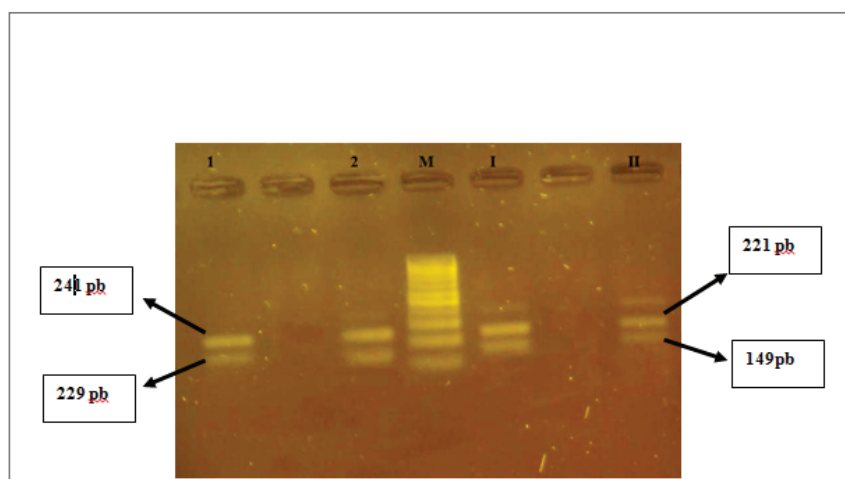

Figure 3: Agarose gel electrophoresis of RFLP-PCR products of $P$. jirovecii DHPS gene by accl and Haelll.

Lanes: M (100 bp DNA size marker); 1and 2(Case 1 and Case2 aftre restriction by AcclI); I and II (Case 1 and Case 2 after restriction by HaellI). 
two patients had an association of wild genotype and mutant genotype [21]. However, Besser, et al. did not detect any mutation in the DHPS gene from Swedish patients. Likewise, we did not detect any mutation in the DHPS gene in both patients and an improvement in the general condition of patients after 14 days of treatment [11]. The absence of mutations is the result of the use of Sulfamethoxazole/Trimethoprim only as first-line treatment for PJP [11,22].

\section{Conclusion}

PJP is a severe, opportunistic respiratory infection that can complicate the course of many immunosuppression-related diseases. In summary, our results show that real-time PCR is more sensitive than conventional techniques which could be added to the diagnostic arsenal of every specialized laboratory. On the other hand, the frequency of DHPS mutations may be higher if the study was conducted on HIV patients. Indeed, their current exposure to sulfonamides can induce resistance to these molecules.

\section{Conflict of Interest}

The authors declare that they have no conflict of interest.

\section{References}

1. Roux A, Gonzalez F, Roux M, Mehrad M, Menotti J, et al. (2014) Update on pulmonary Pneumocystis jirovecii infection in non-HIV patients. Med Mal Infect 44: 185-198.

2. Gaborit BJ, Tessoulin B, Lavergne RA, Morio F, Sagan C, et al. (2019) Outcome and prognostic factors of Pneumocystis jirovecii pneumonia in immunocompromised adults: a prospective observational study. Ann Intensive Care 9: 131.

3. Chatelanat O, Delden CV, Adler D, Guerne PA, Nendaz M, et al. (2018) Risk factors and prophylaxis of Pneumocystis jirovecii pneumonia in HIV-negative patients. Rev Med Suisse 14: 1829-1833.

4. Bossart S, Mühlethaler K, Garzoni C, Furrer H (2020) Is real time PCR preferable to the direct immunofluorescence in the diagnosis of Pneumocystis jirovecii pneumonia in HIV-infected patients? BMC Res Notes 13: 235.

5. Church DL, Ambasta A, Wilmer A, Williscroft H, Ritchie G, et al. (2015) Development and validation of a Pneumocystis jirovecii realtime polymerase chain reaction assay for diagnosis of Pneumocystis pneumonia. Can J Infect Dis Med Microbiol 26: 263-267.

6. Kaouecha E, Kallel K, Ananea S, Belhadja S, Abdellatif, et al. (2009) Pneumocystis jiroveci pneumocystosis: comparative study of PCR and staining techniques. Pathol Biol 57: 373-377.

7. Irinyi L, Hu Y, Hoang MTV, Pasic L, Halliday C, et al. (2020) Longread sequencing based clinical metagenomics for the detection and confirmation of Pneumocystis jirovecii directly from clinical specimens: A paradigm shift in mycological diagnostics. Med Mycol 58: 650-660.

8. Rostved AA, Sassi M, Kurtzhals JAL, Sørensen SS, Rasmussen A, et al. (2013) Outbreak of pneumocystis pneumonia in renal and liver transplant patients caused by genotypically distinct strains of Pneumocystis jirovecii. Transplantation 96: 834-842.
9. Matsumura $Y$, Tsuchido $Y$, Yamamoto M, Nakano S, Nagao M (2019) Development of a fully automated PCR assay for the detection of Pneumocystis jirovecii using the GENECUBE system. Med Mycol 57: 841-847.

10. Fauchier T, Hasseine L, Gari-Toussaint M, Casanova V, Marty PM, et al. (2016) Detection of Pneumocystis jirovecii by Quantitative PCR To Differentiate Colonization and Pneumonia in Immunocompromised HIV-Positive and HIV-Negative Patients. J Clin Microbiol 54: 14871495.

11. Kronbichler A, Kerschbaum J, Gopaluni S, Tieu J, Alberici F, et al. (2018) Trimethoprim-sulfamethoxazole prophylaxis prevents severe/ life-threatening infections following rituximab in antineutrophil cytoplasm antibody-associated vasculitis. Ann Rheum Dis 77: 14401447.

12. Szydłowicz M, Jakuszko K, Szymczak A, Piesiak P, Kowal A, et al. (2019) Prevalence and genotyping of Pneumocystis jirovecii in renal transplant recipients-preliminary report. Parasitol Res 118: 181-189.

13. Carmona EM, Limper AH (2011) Update on the diagnosis and treatment of Pneumocystis pneumonia. Ther Adv Respir Dis 5: 4159.

14. Borstnar S, Lindic J, Tomazic J, Kandus A, Pikelj A, et al. (2013) Pneumocystis jirovecii pneumonia in renal transplant recipients: a national center experience. Transplant Proc 45: 1614-1617.

15. Kostakis ID, Sotiropoulos GC, Kouraklis G (2014) Pneumocystis jirovecii pneumonia in liver transplant recipients: a systematic review. Transplant Proc 46: 3206-3208.

16. Iriart X, Bouar ML, Kamar N, Berry A (2015) Pneumocystis Pneumonia in Solid-Organ Transplant Recipients. J Fungi (Basel) 1: 293-331.

17. Maruschke M, Riebold D, Holtfreter MC, Sombetzki M, Mitzner S, et al. (2014) Pneumocystis pneumonia (PCP) and Pneumocystis jirovecii carriage in renal transplantation patients: a single-centre experience. Wien Klin Wochenschr 126: 762-766.

18. Magne $D$, Angoulvant A, Botterel F, Bouges-Michel $C$, Bougnoux ME, et al. (2009) Pneumocystosis network in lle-de-France: assessment of five years of surveillance (2003-2007). J Med Mycol 19: 290-293.

19. Lee SH, Huh KY, Joo DJ, Kim MS, Kim SI, et al. (2017) Risk factors for Pneumocystis jirovecii pneumonia (PJP) in kidney transplantation recipients. Sci Rep 7: 1-8.

20. Verhaert M, Blockmans D, De Langhe E, Henckaerts L (2020) Pneumocystis jirovecii pneumonia in patients treated for systemic autoimmune disorders: a retrospective analysis of patient characteristics and outcome. Scand J Rheumatol 49: 345-352.

21. Jarboui MA, Sellami A, Sellami H, Cheikhrouhou F, Makni F, et al. (2011) Dihydropteroate synthase gene mutations in Pneumocystis jiroveci strains isolated from immunocompromised patients. Pathol Biol (Paris) 59: 222-225.

22. Ozkoc S, Erguden C, Delibas SB (2018) Absence of dihydropteroate synthase gene mutations in Pneumocystis jirovecii strains isolated from Aegean region of Turkey. Parasitol Res 117: 3103-3108. 